\title{
Impaired circadian heart rate variability in Parkinson's disease: a time-domain analysis in ambulatory setting
}

\author{
V. Arnao ${ }^{1}$, A. Cinturino ${ }^{1}$, S. Mastrilli' ${ }^{1}$ C. Buttà ${ }^{2}$, C. Maida ${ }^{2}$, A. Tuttolomondo ${ }^{2}$, P. Aridon ${ }^{1}$ and M. D'Amelio ${ }^{1 *}$
}

\begin{abstract}
Background: Heart rate variability (HRV) decreases in Parkinson's disease (PD) and it can be considered a marker for cardiovascular dysautonomia. The purpose of this pilot study is to evaluate long-term time-domain analysis of HRV of PD patients and compare the results with those of matched healthy individuals.

Methods: Idiopathic PD patients without comorbidity impairing HRV, and age-matched healthy individuals were recruited in a pilot study. A long-term time domain analysis of HRV using 24-h ambulatory ECG was performed.

Results: Overall, 18 PD patients fulfilling inclusion criteria completed the evaluation (mean age was $55.6 \pm 8.8$, disease duration: $5.0 \pm 4.7$ ). Mean SCOPA-AUT score was $10.1 \pm 7.3$. Patients were on Hoehn \& Yahr stage $1-2$ and mean Levodopa Equivalent Dose (LED) was $311 \pm 239$.9. Mean of the 5 -min standard deviation (SD) of R-R intervals distribution (SDNN) for all 5 min segments of the entire recording (ISDNN) was significantly lower in patients compared to controls. ISDNN was significantly different between Parkinson's disease patients and healthy controls.

Conclusions: In our population characterized by mild to moderate disease severity, time-domain assessment of HRV seemed to be a potential tool to characterize cardiovascular dysautonomia. Decrease of ISDNN in PD may reflect an autonomic derangement extending all day and night long.
\end{abstract}

Keywords: Non-motor symptoms, Autonomic disorders, Heart rate variability, SCOPA-AUT

\section{Background}

Heart rate variability (HRV) is decreased in Parkinson's disease (PD) and it could occur early [1] in the course of the disease. HRV can be considered a marker for cardiovascular sympathovagal balance [2]. HRV is a noninvasive widespread tool for studying heart rate regulation in relation to autonomic system. HRV might be evaluated by a short term (usually $5 \mathrm{~min}$ ) ECG recording obtained under controlled standardized conditions and long term analysis [3]. Typically, higher values of HRV reflect better health and studies using HRV evaluation have reported

\footnotetext{
*Correspondence: marco.damelio@unipa.it

'Department of Biomedicine, Neuroscience and Advanced Diagnostics (BiND), University of Palermo, Via Gaetano la Loggia n.1, 90129 Palermo, Italy Full list of author information is available at the end of the article
}

that it declines in patients with PD compared to healthy matched controls, in short and long recordings $[4,5]$. Short-time spectral analysis is often used in PD patients because it is a fast, low-cost and patient-independent measure, with good intra-individual reproducibility for HRV [3-6]. However, it has been observed that patients with normal responses to short-time spectral analysis tests could suffer from impaired HRV evaluated by a long-term time analysis [3]. Time domain analysis by a 24 -h ECG recording could be a valid tool for characterizing cardiac autonomic state in PD and 24-h HRV indices appear to be stable and free of placebo effect [7].

The main aim of our study was to evaluate whether a difference between PD patients without comorbidities impairing long term time-domain HRV and healthy

C C The Author(s). 2020 Open Access This article is licensed under a Creative Commons Attribution 4.0 International License, which permits use, sharing, adaptation, distribution and reproduction in any medium or format, as long as you give appropriate credit to the original author(s) and the source, provide a link to the Creative Commons licence, and indicate if changes were made. The images or other third party material in this article are included in the article's Creative Commons licence, unless indicated otherwise in a credit line to the material. If material is not included in the article's Creative Commons licence and your intended use is not permitted by statutory regulation or exceeds the permitted use, you will need to obtain permission directly from the copyright holder. To view a copy of this licence, visit http://creativecommons.org/licenses/by/4.0/ The Creative Commons Public Domain Dedication waiver (http://creativecommons.org/publicdomain/zero/1.0/) applies to the data made available in this article, unless otherwise stated in a credit line to the data. 
controls exists. Whether a difference was observed, we determined the characteristics of the disease associated with HRV impairment. A time-domain assessment of HRV was performed using a long term recording.

\section{Methods}

\section{Characteristics of the population}

This was an observational, cross-sectional, comparative pilot study carried-out in an ambulatory setting. Nondemented PD patients were consecutively recruited from our department. Patients with suspected atypical or secondary parkinsonism were excluded, as well as those with comorbidities known to influence HRV (hypertension, diabetes, heart diseases, heart failure, myocardial infarction), or those patients taking drugs that are known to reduce HRV (antidepressants, selegeline, anticholinergic, mineral corticoids, beta blockers, calcium blockers, antiarrhythmics). Similar exclusion criteria were used for healthy controls (individuals non affected by Parkinson's disease or other neurological disorders), who were matched 1:1 for sex and age to PD patients. All the participants gave their informed consent to participate in the study. The study was approved by the local ethics committee (Palermo1 v.n.5/13.05.2015) and conducted according to the principles of the Declaration of Helsinki.

During the study period, 127 patients (males, 55\%) were evaluated. Overall, 104 patients were excluded because they did not meet the inclusion criteria $(22 \mathrm{de}-$ mented, 82 for other comorbidities or taking drugs affecting HRV). Five of the final 23 patients invited, refused to participate in the study.

\section{Clinical and ECG evaluation}

Each patient underwent an extensive evaluation including Hoehn \& Yahr stage (H\&Y) [8], Unified Parkinson's Disease Rating Scale (UPDRS) [9], and Scale for
Outcomes in PD for autonomic symptoms (SCOPAAUT) [10]. Medications and comorbidities were recorded, using the Cumulative Illness Rating Scale (CIRS) [11]. Levodopa Equivalent Daily Dose (LED) [12] was also calculated. A long term time domain analysis of heart rate variability (HRV) using 24-h 12-lead ECG recording (at least 18-h ECG), encompassing morning and night hours was performed and evaluated according to the NASPE/ESC Task Force [7] in PD patients and agematched healthy individuals. Both groups were asked to perform normal daily activity excluding intense physical activity. All time domain parameters analysed have been summarized in Table 1 and calculated using "cardioscan II" software (version 11.4.0054a).

\section{Statistics}

Results are expressed as mean $\pm \mathrm{SD}$, with $p \leq 0.05$ considered significant. Analysis of normality was performed with the Shapiro-Wilk W test $(\alpha: 0,05)$. Differences between cases and controls of HRV time domain parameters were calculated by t-test analysis. When a variable of the HRV time domain analysis was found significantly different between cases and controls, its association with explored PD variables was evaluated by Pearson correlation. Furthermore, linear regression analysis investigated for correlations between patient characteristics: age, LED, HY, LEVODOPA, SCOPA-AUT (independent variables) and HRV time domain parameters (dependent variable) in simple and multiple regression models. B coefficients (B) and their 95\% confidence intervals (CIs) were also calculated. The post-hoc statistical power and the effect sizes on the basis of standardized mean differences (Cohen's d) were performed too by Clin.Calc.com (https://ckinical.com/stat/Power-aspx accessed on 01/ 12/2019) and G*Power 3.1.9.3 software to compute statistical power in sample size of our pilot study.

Table 1 HRV Time domain parameters evaluated in PD patients and controls

\begin{tabular}{ll}
\hline Variables & Description (unit) \\
\hline SDNN 24 & Standard deviation (SD) of all NN intervals $(m s)$ \\
SDNN D & \\
SDNN N & \\
pnn50 24 & Percentage of successive RR intervals that differ by more than 50 ms (\%) \\
pnn50 N & \\
rMSS 24 & Root mean square of successive RR interval differences ( $m s)$ \\
rMSS D & \\
rMSS N & \\
SDANN & Standard deviation of the average NN intervals for each 5 min segment of a $24 \mathrm{~h}$ HRV recording ( $m s)$ \\
ISDNN & Mean of the Standard deviation of all NN intervals for all 5 min segments of the entire recording (ms) \\
\hline
\end{tabular}




\section{Results}

Overall, eighteen PD patients (9 males) with a mean disease duration of $50 \pm 4.7$ years were enrolled in this study. No significant difference of age at HRV evaluation was observed between PD patients (mean age 55.6 \pm 8.8 years), and controls (mean age $56.0 \pm 9.4$ years) $(p=0.8)$. PD patients had a mean SCOPA-AUT scale score of 10.1 \pm 7.3. Patients with PD were characterized by mild to moderate disease severity (HY stage 1-2) and mean LED was $311 \pm 239.9$. Only SDNN index (ISDNN), evaluating mean of the standard deviations of all $\mathrm{NN}$ intervals for all $5 \mathrm{~min}$ segments of the entire recording, was significantly different between PD patients (42.9 \pm $14.2 \mathrm{~ms})$ and healthy controls $(53.4 \pm 10.1 \mathrm{~ms}) \quad(p=$ 0.01 )(see Table 2). We plotted ISDNN values for PD patients and controls (see Fig. 1). Statistical power was calculated (power 72. 5\%, $\alpha: 0.05$ ), whereas, observed Cohen'd was 0. 85.

ISDNN significantly correlated with age $(r-0.69, p=$ $0.02)$, LED $(r-0.60, p=0.01)$, levodopa dosage $(r-0.57$, $p=0.02)$ and SCOPA-AUT scale scores $(r-0.47, p=$ $0.05)$. At linear regression analysis in simple regression model, a statistically significant relationship between age, levodopa dosage, HY and LED and ISDNN (see Fig. 2) was observed, but not confirmed in multiple regression model.

\section{Discussion}

In our study, recruiting only non-demented PD patients, with mild to moderate disease severity and no other conditions (comorbidities or treatments) known to affect HRV, ISDNN was significantly different between Parkinson's disease patients and healthy controls. ISDNN seems to correlate with age, severity of disease stage,

Table 2 HRV parameters (means \pm SD) in PD patients and controls

\begin{tabular}{llll}
\hline Variables & PD patients (18) & Controls (18) & $p$ \\
\hline SEX(men\%) & $9(50)$ & $9(50)$ & \\
AGE(years) & $55.6 \pm 8.8$ & $55.6 \pm 8.8$ & 0.8 \\
SDNN 24(ms) & $122.6 \pm 38.3$ & $136.0 \pm 21.4$ & 0.2 \\
SDNN D(ms) & $84.3 \pm 27.9$ & $96.2 \pm 36.5$ & 0.3 \\
SDNN N(ms) & $112.6 \pm 40.9$ & $119 \pm 31.3$ & 0.6 \\
pnn50 24(\%) & $4.5 \pm 4.1$ & $5.3 \pm 5.3$ & 0.6 \\
pnn50 D(\%) & $2.5 \pm 2.3$ & $3.8 \pm 4.8$ & 0.3 \\
pnn50 N(\%) & $9.3 \pm 9.4$ & $8.4 \pm 7.6$ & 0.8 \\
rMSS 24(ms) & $24.9 \pm 7$ & $25.9 \pm 7.7$ & 0.7 \\
rMSS D(ms) & $20.8 \pm 5.5$ & $23.4 \pm 8.9$ & 0.3 \\
rMSS N (ms) & $31.1 \pm 11.9$ & $29.6 \pm 9.5$ & 0.7 \\
SDANN(ms) & $118.1 \pm 38.8$ & $124.7 \pm 21.2$ & 0.5 \\
ISDNN(ms) & $42.9 \pm 14.2$ & $53.4 \pm 10.1$ & 0.01 \\
\hline PD Parkins's dseas & & &
\end{tabular}

PD Parkinson's disease; $D$ day; $N$ night
LED and levodopa dosages. Taking into account the task force on the HRV, SDNN, reflecting over heart variability, is not a well-defined statistical variable due to its dependence on ECG time recording, while ISDNN, evaluating too over heart variability, is a more standardized variable [7].

ISDNN most closely correlates with age, declining with aging; gender also influences HRV (women had lower values); however in our study we enrolled PD patients and healthy controls matched for age and sex [13].

In a recent study, including $1.741 \mathrm{PD}$ patients (ECG recording has been available for 653 PD patients), no association was reported between HRV (SDNN and rMSS) and PD severity or its progression [14], although routine ECG data (standard 10-s 12-lead ECG) was used to perform HRV; standard ECG recording is considered inadequate for measuring HRV. According to the task force on the HRV, short-term 5-min recordings and nominal 24-h long-term recordings seem to be appropriate options. Moreover, patients with significant comorbidities or those taking drugs impairing HRV were not excluded. ISDNN, the mean of the 5-min standard deviation of the $\mathrm{NN}$ interval calculated over $24 \mathrm{~h}$, which measures the variability due to cycles shorter than $5 \mathrm{~min}$, requires a 24-h ECG recording. Both cardiac sympathetic and parasympathetic dysfunctions are commonly reported in PD [15] ISDNN primarily reflects autonomic influence on heart rate variability [16]. Decrease of ISDNN, reflecting over variability of HRV, may be demonstrated in PD by a sympathovagal balance impairment. A body of evidence has challenged the traditional view of $\mathrm{PD}$ as a motor disorder, in favour of the idea of PD being a more complex disorder, including non-motor symptoms [17]. Among non-motor symptoms, autonomic dysfunction is frequent in PD patients $[18,19]$ with cardiovascular autonomic dysfunction occurring early in the course of the disease [20]. ISDNN, evaluating HRV in $24 \mathrm{~h}$, implies a more complex derangement extending all day and all night and it could reflect an impairment of diurnaltemporal component; suggesting an imbalance of circadian system as observed for other non-motor symptoms in PD. The balance between sympathetic and parasympathetic varies in synchrony with the circadian system [21]. Circadian dysfunction could be part of the new view of PD highlighting the role of non-motor symptoms and its temporal pattern. Our pilot study was characterized by strengths and limitations. The major strength of our study was the inclusion of individuals (patients and healthy controls) without comorbidities or taking drugs known to affect HRV. The observed difference in HRV between the two groups was therefore hypothetically almost exclusively due to the presence of PD. Other strengths of our study were the strict matching for age and sex between cases and controls, as well as the use of 


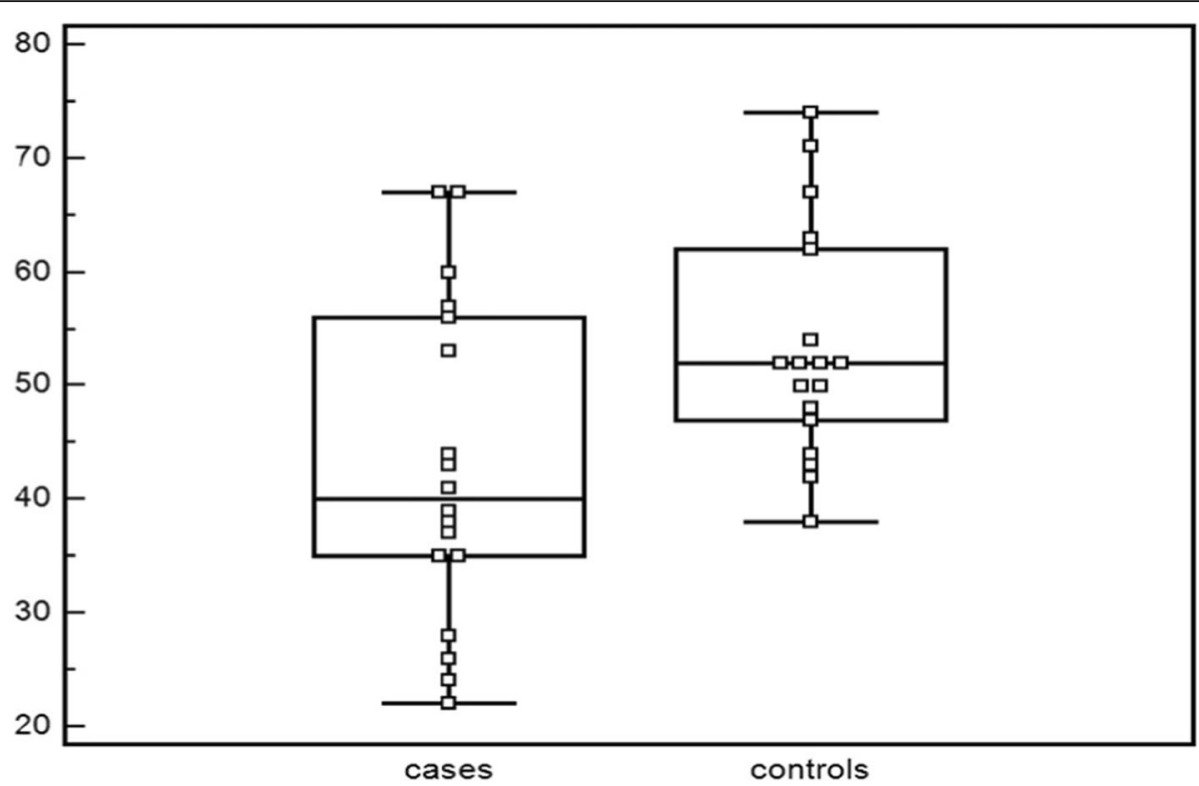

Fig. 1 ISDNN mean and standard deviations in PD patients and controls

a validated scale, the SCOPA-AUT, in order to detect the presence of autonomic dysfunction. The main limitation of our pilot study was the small sample size, attributable to the decision to adopt rigid exclusion criteria including concomitant medical issues and the use of some drugs. This led to the elimination of nearly $65 \%$ of the patients. It is presumable that, in an ambulatory setting, autonomic evaluation would be in the near future considered together with motor and other non-motor examination. The evaluation should include a questionnaire such as the SCOPA-AUT scale, and tools such as the long-term time-domain assessment of HRV; as lowcost and fast evaluations have to be fostered, our findings suggest that, in an ambulatory setting, the use of HRV analyses could be considered as part of an instrumental evaluation for the detection of autonomic dysfunction. This is in fact a promising, simple, non- invasive and low-cost test for cardiovascular autonomic symptoms in PD patients.

\section{Conclusions}

Autonomic symptoms are common but often unrecognized in patients with Parkinson's disease. In our population characterized by mild to moderate disease severity, time-domain assessment of HRV seemed to be a potential tool for characterizing cardiovascular dysautonomia. In our pilot study, ISDNN was significantly different between PD patients and matched healthy controls. In an ambulatory setting, according to our findings, the use of HRV analysis could be considered as part of an instrumental evaluation for cardiovascular autonomic symptoms in PD patients.
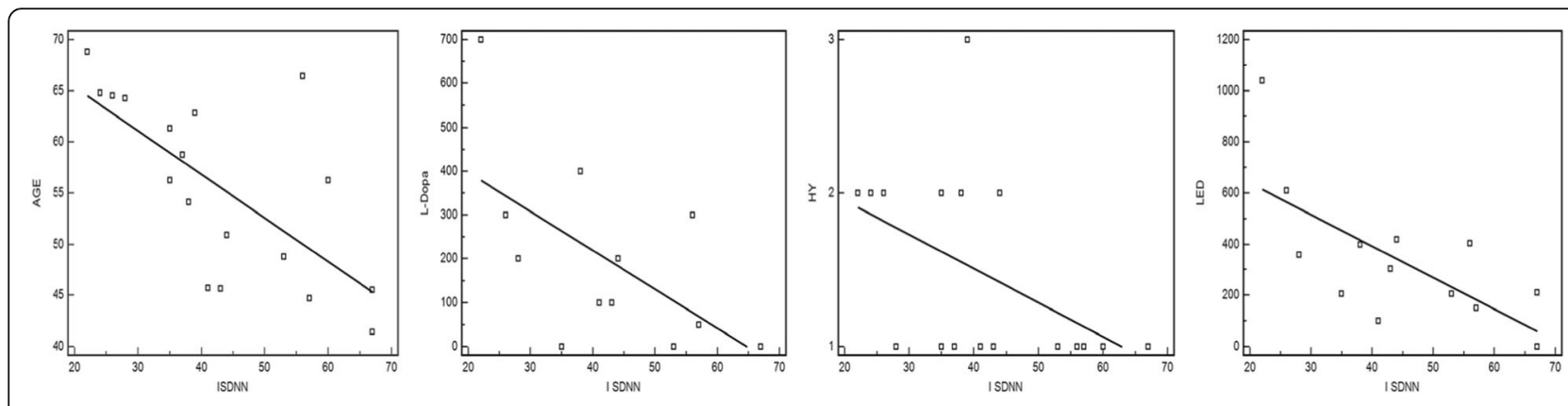

Fig. 2 correlation by linear regression between ISDNN and clinical PD variables (AGE, Levodopa dosage, HY, LED) 


\section{Abbreviations}

CIRS: Cumulative Illness Rating Scale; D: Day; HRV: Heart rate variability; H\&Y: Hoehn \& Yahr stage; ISDNN: Mean of the SD of all NN intervals for all 5 min segments of the entire recording; LED: Levodopa Equivalent Dose; N: Night; PD: Parkinson's disease; pNN50: NN50 count divided by the total number of all NN intervals; r-MSSD: Square root of the mean of the sum of the squares of differences between adjacent NN Intervals; SCN: suprachiasmatic nuclei; SCOPA-AUT: Scale for Outcomes in PD for autonomic symptoms; SD: Standard deviation; SDNN: Standard deviation of all NN intervals; UPDRS: Unified Parkinson's Disease Rating Scale

\section{Acknowledgements}

Not applicable.

\section{Authors' contributions}

VA participated in the conception and design of the study, as well as the acquisition, analysis and interpretation of the data and the writing of the first draft of the manuscript and its critical revision. AC participated in the conception and design of the study, along with the acquisition of data, and the drafting and critical revision of the manuscript. SM participated in the acquisition of the data. CB participated in the interpretation of the data. CM participated in the interpretation of the data. AT participated in the interpretation of the data and the drafting the article and its critical revision. PA participated in the interpretation of the data and in the critical revision of the article. MD participated in the design of the study, as well as the acquisition, analysis and interpretation of data, along with contributing to the drafting of the manuscript and its critical revision. All Authors have read and approved the manuscript.

\section{Funding}

Not applicable.

\section{Availability of data and materials}

The datasets used and/or analysed during the current study are available from the corresponding author on reasonable request.

\section{Ethics approval and consent to participate}

The local Ethics Committee "Comitato Etico Palermo 1 AUOP Paolo Giaccone" approved the experimental protocol (Verbale N5/13.05.2015). It was performed in accordance with the ethical standards as laid down in the 1964 Declaration of Helsinki and its later amendments. Written informed consent was obtained from all participants.

\section{Consent for publication}

Not applicable.

\section{Competing interests}

The authors declare that they have no competing interests.

\section{Author details}

'Department of Biomedicine, Neuroscience and Advanced Diagnostics (BiND), University of Palermo, Via Gaetano la Loggia n.1, 90129 Palermo, Italy. 2Dipartimento Biomedico di Medicina Interna e Specialistica (Di.Bi.M.I.S), Università degli Studi di Palermo, Palermo, Italy.

Received: 7 October 2019 Accepted: 12 April 2020

Published online: 23 April 2020

\section{References}

1. Barbic F, Perego F, Canesi M, Gianni M, Biagiotti S, Costantino G, et al. Early abnormalities of vascular and cardiac autonomic control in Parkinson's disease without orthostatic hypotension. Hypertension. 2007. https://doi. org/10.1161/01.HYP.0000250939.71343.7C.

2. Mastrocola C, Vanacore N, Giovani A, Locuratolo N, Vella C, Alessandri A, et al. Twenty-four-hour heart rate variability to assess autonomic function in Parkinson's disease. Acta Neurol Scand. 1999. https://doi.org/10.1111/j.16000404.1999.tb07355.x

3. Cygankiewicz I, Zareba W. Heart rate variability. Handb Clin Neurol. 2013. https://doi.org/10.1016/B978-0-444-53491-0.00031-6.

4. Haapaniemi TH, Pursiainen V, Korpelainen JT, Huikuri HV, Sotaniemi KA, Myllylä W. Ambulatory ECG and analysis of heart rate variability in
Parkinson's disease. J Neurol Neurosurg Psychiatry. 2001. https://doi.org/10. 1136/jnnp.70.3.305.

5. Katagiri A, Asahina M, Araki N, et al. Myocardial (123)I-MIBG uptake and cardiovascular autonomic function in Parkinson's disease. Parkinsons Dis. 2015. https://doi.org/10.1155/2015/80535.

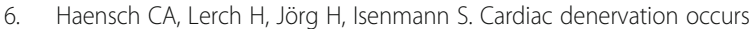
independent of orthostatic hypotension and impaired heart rate variability in Parkinson's disease. Parkinsonism Relat Disord. 2009. https://doi.org/10. 1016/j.parkreldis.2008.04.031.

7. Heart rate variability. Standards of measurement, physiological interpretation, and clinical use. Task Force of the European Society of Cardiology and the North American Society of Pacing and Electrophysiology 1996. Eur Heart J;7:354-81..

8. Hoehn MM, Yahr MD. Parkinsonism: onset, progression and mortality. Neurology. 1967. https://doi.org/10.1212/wnl.17.5.427.

9. Fahn S, Marsden CD, Calne DB, Goldstein M. (eds): recent developments in Parkinson's disease. Florham Park. Macmillan Healthcare Information. 1987;2: 153-63.

10. Visser M, Marinus J, Stiggelbout AM, Van Hilten JJ. Assessment of autonomic dysfunction in Parkinson's disease: the SCOPA-AUT. Mov Disord. 2004. https://doi.org/10.1002/mds.20153.

11. Linn BS, Linn MW, Gurel L. Cumulative illness rating scale. J Am Geriatr Soc. 1968. https://doi.org/10.1111/j.1532-5415.1968.tb02103.x.

12. Tomlinson CL, Stowe $R$, Patel S, Rick C, Gray R, Clarke CE. Systematic review of levodopa dose equivalency reporting in Parkinson's disease. Mov Disord. 2010. https://doi.org/10.1002/mds.23429.

13. Umetani K, Singer DH, McCraty R, Atkinson M. Twenty-four hour time domain heart rate variability and heart rate: relations to age and gender over nine decades. J Am Coll Cardiol. 1998. https://doi.org/10.1016/s07351097(97)00554-8.

14. Gibbons $\mathrm{CH}$, Simon DK, Huang M, Tilley B, Aminoff MJ, Bainbridge $\mathrm{J}$, et al. Autonomic and electrocardiographic findings in Parkinson's disease. Auton Neurosci. 2017. https://doi.org/10.1016/j.autneu.2017.04.002.

15. Jain S. Multi-organ autonomic dysfunction in Parkinson disease. Parkinsonism Relat Disord. 2011. https://doi.org/10.1016/j.parkreldis.2010.08. 022.

16. Shaffer F, Ginsberg JP. An overview of heart rate variability metrics and norms. Front Public Health. 2017. https://doi.org/10.3389/fpubh.2017.00258.

17. Kalia LV, Lang AE. Parkinson's disease. Lancet. 2015. https://doi.org/10.1016/ S0140-6736(14)61393-3.

18. Arnao V, Cinturino A, Valentino F, Perini V, Mastrilli S, Bellavia G, et al. In patient's with Parkinson disease, autonomic symptoms are frequent and associated with other non-motor symptoms. Clin Auton Res. 2015. https:// doi.org/10.1007/s10286-015-0306-x

19. De Pablo-Fernandez E, Tur C, Revesz T, Lees AJ, Holton JL, Warner TT. Association of Autonomic Dysfunction with Disease Progression and Survival in Parkinson disease. JAMA Neurol. 2017. https://doi.org/10.1001/ jamaneurol.2017.1125

20. Valappil RA, Black JE, Broderick MJ, Carrillo O, Frenette E, Sullivan SS. Exploring the electrocardiogram as a potential tool to screen for premotor Parkinson's disease. Mov Disord. 2010. https://doi.org/10.1002/mds.23348.

21. Willison LD, Kudo T, Loh DH, Kuljis D, Colwell CS. Circadian dysfunction may be a key component of the non-motor symptoms of Parkinson's disease: insights from a transgenic mouse model. Exp Neurol. 2013. https://doi.org/ 10.1016/j.expneurol.2013.01.014.

\section{Publisher's Note}

Springer Nature remains neutral with regard to jurisdictional claims in published maps and institutional affiliations. 